\title{
Differential Roles of Water-Insoluble and -Soluble Fractions of Diesel Exhaust Particles in the Development of Adverse Health Effects due to Chronic Instillation of Diesel Exhaust Particles
}

\section{Yanyi Xu}

Fudan University School of Public Health

Zhouzhou Li

Fudan University School of Public Health

Ying Liu

Clinical Research Center

\section{Bin Pan}

Fudan University School of Public Health

\section{Renzheng Peng}

Fudan University School of Public Health

Wenpu Shao

Fudan University School of Public Health

\section{Wenhui Yang}

Fudan University School of Public Health

\section{Minjie Chen}

University of Maryland School of Medicine

\section{Haidong Kan}

Fudan University School of Public Health

\section{Zhekang Ying}

University of Maryland, Baltimore

Yuhao Zhang ( $\nabla$ zhang.yuhao@zs-hospital.sh.cn )

Zhongshan Hospital Fudan University

\section{Research}

Keywords: PM2.5, solubility, glucose intolerance, insulin resistance, pulmonary inflammation, hepatic steatosis

Posted Date: May 27th, 2021

DOI: https://doi.org/10.21203/rs.3.rs-527024/v1

License: @ (i) This work is licensed under a Creative Commons Attribution 4.0 International License. Read Full License

Version of Record: A version of this preprint was published at Chemical Research in Toxicology on November 15th, 2021. See the published version at https://doi.org/10.1021/acs.chemrestox.1c00199. 


\section{Abstract}

Background: Ambient fine particulate matter $\left(\mathrm{PM}_{2.5}\right)$ has a marked temporospatial variation in chemical composition, but how the composition of $\mathrm{PM}_{2.5}$ influences its toxicity remains elusive.

Results: To explore the individual roles of different $\mathrm{PM}_{2.5}$ components in the pathogenesis due to $\mathrm{PM}_{2.5}$ exposure, we prepared water-soluble (WS-DEP) and -insoluble (WIS-DEP) fractions of diesel exhaust particles (DEP) and assessed their effects on pulmonary and systemic inflammation, hepatic steatosis and insulin resistance, systemic glucose homeostasis, and gut microbiota using chronic intratracheal instillation mouse models. Compared to control, instillation of DEP or WIS-DEP, but not WS-DEP, significantly increased pulmonary inflammatory scores and expression of inflammatory markers, bronchoalveolar lavage fluid cell number, and circulating pro-inflammatory cytokines. Consistently, DEP or WIS-DEP-instilled but not WS-DEP-instilled mice versus control had significant hepatic steatosis and insulin resistance and systemic glucose intolerance. In contrast, instillation of WS-DEP versus instillation of WISDEP had more similar effects on gut microbiota to that of instillations of DEP.

Conclusion: The pulmonary and systemic inflammation, hepatic steatosis and insulin resistance, and systemic glucose intolerance following chronic DEP instillation are all attributable to the water-insoluble fraction of DEP, providing a mechanistic interpretation for the apparent independency of $\mathrm{PM}_{2.5}$ exposure-induced glucose intolerance on $\mathrm{PM}_{2.5}$ composition.

\section{Background}

Fine particulate matter $\left(\mathrm{PM}_{2.5}\right)$ is the fraction of particles with aerodynamic diameters $\leq 2.5 \mu \mathrm{m}$. This arrange of aerodynamic diameters allow particles to enter the airway deeply and thus likely deposit in the alveoli where the cleaning of particles is very slow due to the lack of the mucociliary escalator. A huge number of epidemiological and toxicological studies have shown that inhalation of $\mathrm{PM}_{2.5}$ impacts not only the lung but also many other major organs/tissues (e.g., the liver) and subsequently provokes systemic pathologies (e.g., abnormal glucose homeostasis). $(1,2)$ The most recent global disease burden analysis clearly demonstrated that $\mathrm{PM}_{2.5}$ pollution is one of the leading threats to the global public health.(1) However, despite the considerable scientific effort over the past decade, the biological mechanism for the development of systemic health effects following $\mathrm{PM}_{2.5}$ inhalation remains elusive. This is at least partly attributable to the temporospatial variation in $\mathrm{PM}_{2.5}$ chemical composition. As mentioned above, $\mathrm{PM}_{2.5}$ is defined per the aerodynamic diameter. Therefore, its chemical composition may vary largely, and thus there is also a focused scientific effort over the last decade to determine the components of $\mathrm{PM}_{2.5}$ that are more harmful than others.

Mounting epidemiological studies over the world, however, have collectively shown that although organic components, sulfate, and some elements (e.g., Ni, V, Zn, Cu, Si, Al, and K) of $\mathrm{PM}_{2.5}$ are more frequently associated with adverse health responses to $\mathrm{PM}_{2.5}$ exposure, no any major component of $\mathrm{PM}_{2.5}$ can yet be dismissed as being unequivocally not associated with adverse health responses.(3) Interestingly, a series of toxicological studies suggest that Ni may be a potential putative factor in health effects induced by $\mathrm{PM}_{2.5}$ exposure.(4) However, these studies are performed in New York only, and thus it is unclear whether this is applied to $\mathrm{PM}_{2.5}$ in other areas. Indeed, several other elements (e.g., V, Fe, $\mathrm{Cr}$, and $\mathrm{Al}$ ) are also frequently indicted for the adverse health effects induced by $\mathrm{PM}_{2.5}$ exposure in other toxicological studies.(3) Notably, we previously showed that ambient $\mathrm{PM}_{2.5}$ from Baltimore, Maryland, ${ }^{(5)}$ Tuxedo, New York, ${ }^{(6)}$ and Shanghai, China ${ }^{(7)}$ had distinct chemical compositions, representing those of ambient $\mathrm{PM}_{2.5}$ in urban, rural, and construction areas, respectively, but exposures to these $\mathrm{PM}_{2.5}$ all elicited marked systemic insulin resistance and 
glucose intolerance. ${ }^{(7-10)}$ All these strongly suggest that there may not be any putative individual chemical that is primarily responsible for the systemic health effects of $\mathrm{PM}_{2.5}$.

In addition to the above effort to seek individual chemicals of $\mathrm{PM}_{2.5}$ that may be indicted for the adverse health effects of $\mathrm{PM}_{2.5}$ exposure, mounting studies have investigated the role of grouped chemicals in the development of adverse health effects due to $\mathrm{PM}_{2.5}$ exposure. $(3,11)$ For example, several epidemiological studies have examined whether

$\mathrm{PM}_{2.5}$ sources correlate to the toxicity of $\mathrm{PM}_{2.5}$, and diesel exhaust comes out to be the most frequently identified culprit for the development of adverse health effects induced by $\mathrm{PM}_{2.5}$ exposure. $(3,11)$ The water solubility may determine the access of $\mathrm{PM}_{2.5}$ components to the systemic circulation and thus their modes of action in the development of systemic health effects.(11) As such, the water solubility-based grouping of $\mathrm{PM}_{2.5}$ components is likely able to fractionate the toxicity of $\mathrm{PM}_{2.5}$. Unexpectedly, few published studies have ever investigated the individual roles of water-soluble and -insoluble fractions of $\mathrm{PM}_{2.5}$ in the development of systemic health effects due to chronic exposure to $\mathrm{PM}_{2.5}$. Therefore, the present study prepared the water-soluble and insoluble fractions of diesel exhaust particles (DEP) at a concentration equivalent to that of DEP and then compared their chronic health effects in C57BI/6J mice.

\section{Methods}

\section{Intratracheal instillation}

The DEP powder was purchased from the National Institute of Standards and Technology (SRM 2975). To obtain the water-soluble (WS-DEP) and -insoluble (WIS-DEP) fractions at a concentration equivalent to that of DEP, the DEP powder was suspended in sterile phosphate-buffered saline (PBS, Fisher scientific) at a concentration of $2 \mathrm{mg} / \mathrm{ml}$, vortexed for $30 \mathrm{~min}$, and then centrifuged for $30 \mathrm{~min}$ at 12,000 g. The supernatant was transferred to a new vial and adjusted to the same volume as the initial suspension using sterile PBS. The pellet was also suspended in the same volume as the initial suspension using sterile PBS.

Male C57BI/6J mice (3-week-old) were purchased from the Animal Center of Shanghai Medical School, Fudan University. After 1 week of acclimation, 10/group mice were randomized into four groups and intratracheally instilled with PBS, DEP, WS-DEP, or WIS-DEP, 3 times per week (Monday, Wednesday, and Friday), and for 15 weeks in total. To minimize the aggregation of particles, suspensions were sonicated (Clifton Ultrasonic Bath) for 20 min and vortexed for $30 \mathrm{~s}$ before each instillation. Instillation was performed as described previously.(12) In brief, mice were anesthetized with $3 \%$ isoflurane, and placed supine with extended neck on an angled board. An 18-gauge cannula (Becton Dickinson) was inserted into the trachea. Suspensions (50 $\mu$ I PBS, WS-DEP, WIS-DEP, or DEP) were then intratracheally administrated via a sterile syringe, followed by an air bolus of $150 \mu \mathrm{l}$. The incubation catheter was removed, and the mouse was transferred to a vertical hanging position with the head up for $5 \mathrm{~min}$, to make sure that the delivered materials were maintained in the lung. During the whole experimental period, all mice were housed in the animal facilities of Fudan University, which maintained a $12 \mathrm{~h}$ light/ $12 \mathrm{~h}$ dark cycle, a room temperature of $20-25^{\circ} \mathrm{C}$, and a relative humidity of $40-70 \%$. All animal-related procedures were approved by the institutional animal care and use committees of Fudan University, and all the animals were treated humanely and with regard for alleviation of suffering.

\section{Intraperitoneal glucose tolerance test (IPGTT) and insulin tolerance test (ITT)}

IPGTT was performed after 12 weeks of instillation. Briefly, after a 16-hour fasting, the basal blood glucose levels of mice were determined using an automatic glucometer (Glucotrend 2, Roche Diagnostics). The mice were then intraperitoneally injected with glucose $(2 \mathrm{~g} / \mathrm{kg}$ of body weight) and the blood glucose levels at 15, 30, 60, and $120 \mathrm{~min}$ 
after injection of glucose were measured as described above. ITT was performed after 13 weeks of instillation. Briefly, after a 4-hour fasting, the basal blood glucose levels of mice were determined using an automatic glucometer (Glucotrend 2, Roche Diagnostics). The mice were then intraperitoneally injected with insulin (0.5 U/kg of body weight), and the blood glucose levels at 15, 30,60, and 120 min after injection of insulin were measured as described above.

\section{Fecal sample collection, DNA extraction and sequencing}

After 14 weeks of instillation, mice were transferred to clean cage without any bedding (individually housed) and allowed to defecate normally. 24- $\mathrm{h}$ fecal pellets of each mouse were transferred to sterile Eppendorf tubes and stored at $-80{ }^{\circ} \mathrm{C}$ until ready to extract DNA. The total genomic DNA of fecal sample was extracted using a MoBio PowerFecal DNA extraction kit (Qiagen) per the manufacturer's instructions. In brief, samples were firstly homogenized in a tube with garnet beads. The lysis of microbial cells and host cells was facilitated by both chemical disruption of cell membranes and mechanical collisions between beads to ensure efficient extraction. The extracted genomic DNA was captured in a silica spin column and eluted out with elution buffer $(50 \mu \mathrm{l})$. To generate the sequencing libraries, the concentration and quality of extracted DNA was assessed using a NanoDrop 1000 spectrophotometer (Thermo Scientific) and agarose gel electrophoresis (1\% wt/vol agarose in tris-acetate-EDTA buffer) respectively. The bacterial 16s rRNA V4 region was amplified by PCR using the barcoded primers: 515F (5'-GTG CCA GCM GCC GCG G-3') and 907R (5'-CCG TCA ATT CMT TTR AGT TT-3'). Thermal cycling was performed using a 9700 PCR system (ABI, GeneAmp 9700), and all the PCR products were subjected to agarose gel electrophoresis (2\%) followed by purification using the AXYGEN gel extraction kit (Axygen). The purified amplicons were quantified using the Quant-iT PicoGreen dsDNA Assay kit (Thermo Fisher) and QuantiFluor ${ }^{\mathrm{TM}}$-ST Blue-florescence quantitative system (Promega), and then sequenced using the Illumina MiSeq system (Illumina) per the manufacturer's instructions.

\section{Bioinformatics analysis and bacterial diversity and richness analysis}

The primary sequencing data were saved in Fastq format at SRA (Sequence Archive, http://www.ncbi.nlm.nih.gov/Traces/sra). All pyrosequencing reads were pre-processed based on the barcode and primer-end readers using the Usearch software (http://drive5.com/uparse/). All recruited reads had the barcode, a minimal average quality score of 20 , and a maximum of 2 mismatches within the primers. All the overlapped reads with a minimal overlap of $10 \mathrm{bps}$ with a mismatched rate $\leq 0.2$ were merged. The repetitive sequences were extracted and discarded (http://drive5.com/usearch/manual/singletons.html) to avoid unnecessary computations. The optimized sequences were clustered into operational taxonomic unit (OTU) with UCLUST followed by de novo OTU picking. The chimeras were removed using RDP gold database of Usearch software (http://drive5.com/uparse/). The bacterial taxonomy was assigned via the Naïve Bayesian classifier in QIIME platform using the SILVA database (Release 119, http://www.arbsilva.de).

The a-diversity estimators including ACE, CHAO-1, Shannon, and Simpson were obtained using matrices of Mothur (www.mothur.org/wiki/ Schloss_SOP\#Alpha_diversity). ACE and Chao-1 are quantitative indicators of the richness of a sample, and Shannon and Simpson are quantitative indicators of biodiversity (a smaller Shannon estimator or a larger Simpson estimator indicates a lower community diversity). The $\beta$-diversity of bacterial community was assessed by UniFrac distance analysis, principal component analysis (PCA), and principal co-ordinates analysis (PCOA) using the relative abundance of OTU. The linear discriminant effect size (LEfSe) analysis was performed to find OTUs differentially represented in-between the experimental groups: a nonparametric factorial Kruskal-Wallis sum-rank test was used to detect significantly $(p<0.05)$ differential taxa, and the identified taxa were subjected to a linear discriminant analysis (LDA) to evaluate the effect size of each single differential taxon.

\section{Mouse euthanasia and tissue harvesting}

Page 4/21 
Mice were fasted overnight and assessed for body weight and head-to-tail length on the experiment day. In 20 min after i.p. injection of insulin $(10 \mathrm{U} / \mathrm{kg})$, the mice were euthanized, and the blood was harvested from the orbital venous plexus. The mouse trachea was then cannulated, and the right primary bronchus was closed off with a ligation. $0.5 \mathrm{~mL}$ of sterile PBS with $0.1 \mathrm{mM}$ EDTA was instilled through the tracheal cannula and withdrawn to recover the bronchoalveolar lavage fluid (BALF). This process was repeated for three times. The cell number in BALF was determined using a hemocytometer and the protein in BALF was quantified by the bicinchoninic acid assay (BCA, Biyotime) per the manufacturer's instruction. The ligation of right primary bronchus was removed after harvesting BALF and the right lung was ligated in the middle. The lower half was fixed using $4 \%$ paraformaldehyde through the trachea and the upper half was snap-frozen in liquid nitrogen and stored at $-80{ }^{\circ} \mathrm{C}$. Other tissues including brain, heart, liver, kidney, pancreas, spleen, testis, epididymis, skeletal muscle, perirenal adipose tissue, epididymal adipose tissue, subcutaneous adipose tissue, and brown adipose tissue were harvested, weighted, and fixed in $4 \%$ paraformaldehyde for morphological analysis and/or snap-frozen in liquid nitrogen and then stored at $-80^{\circ} \mathrm{C}$ for further processing.

\section{Biochemical analysis}

Levels of aspartate transaminase (AST, Mouse AST Elisa Kit, JianCheng Bioengineering Institute, Nanjing, China), alanine transaminase (ALT, Mouse ALT Elisa Kit, JianCheng Bioengineering Institute, Nanjing, China), total triglycerides (TG, Mouse TG Elisa Kit, JianCheng Bioengineering Institute, Nanjing, China), total cholesterol (Mouse TC Elisa Kit, JianCheng Bioengineering Institute, Nanjing, China), low density lipoprotein (LDL) cholesterol (Mouse LDL-C Elisa Kit, JianCheng Bioengineering Institute, Nanjing, China), and high density lipoprotein (HDL) cholesterol (Mouse HDL-C Elisa Kit, JianCheng Bioengineering Institute, Nanjing, China) in serum or homogenized liver tissue were determined per manufacturer's instructions.

\section{Quantitative real-time RT-PCR (qPCR)}

Total RNA was isolated from frozen lung tissues with Invitrogen ${ }^{\mathrm{TM}}$ TRIzolTM $^{\mathrm{TM}}$ reagent (Invitrogen) per the manufacturer's instruction. The quality and concentration of RNA was determined using a nanodrop spectrophotometer (ThermoFisher Scientific). $2 \mu \mathrm{g}$ of DNase-treated RNA was then reverse-transcribed to cDNA using a High-Capacity cDNA Reverse Transcription Kit (Applied Biosystem). Real-time RT-PCR was performed using SYBR qPCR Master Mix (Vazyme) with

a 7500 Real-time PCR system (Applied Biosystems). The sequences of PCR primers were as follows: Glyceraldehyde-3phosphate dehydrogenase (GAPDH): 5'-TGA ACG GGA AGC TCA CTG G-3' and 5'-TCC ACC ACC CTG TTG CTG TA-3'; Interleukin (IL)-1ß: 5'-ACG GAC CCC AAA AGA TGA AG-3' and 5'-TTC TCC ACA GCC ACA ATG AG-3'; IL-6: 5'-ATC CAG TTG CCT TCT TGG GAC TGA-3' and 5'-TAA GCC TCC GAC TTG TGA AGT GGT-3'; Tumor necrosis factor alpha (TNFa) 5'-TTC CGA ATT CAC TGG AGC CTC GAA-3' and 5'-TGC ACC TCA GGG AAG AAT CTG GAA-3'. $2^{\Delta C t}$ was calculated as the relative expression level of detected genes as previously described. ${ }^{(13)}$

\section{Histological analysis}

The fixed lung tissue was embedded in paraffin, cut into $5-\mu \mathrm{m}$ sections. 6 consecutive lung sections/mouse were subjected to $\mathrm{H}$ \& $\mathrm{E}$ staining and images covering all lung tissues were taken and analyzed by a pathologist who was blind to the grouping. The pulmonary inflammation level was quantitated per the scoring system in Table 3. An aliquot of liver was fixed at $4{ }^{\circ} \mathrm{C}$ in $4 \%$ paraformaldehyde for 24 hours. These liver tissues were embedded in paraffine and cut into 5 - $\mu \mathrm{m}$ sections. 6 consecutive sections/mouse were subjected to H \& E staining or Masson's trichrome staining and images covering all liver tissues were taken and analyzed by a pathologist who was blind to the grouping. Hepatic steatosis, hepatocyte ballooning, lobular and portal inflammation, Mallory bodies, and fibrosis were examined and scored according to the modified Brunt scoring system for non-alcoholic fatty liver disease. Another aliquot of liver tissue was frozen in optimal cutting temperature compound, cut into $4-\mu \mathrm{m}$ sections, and subjected to Oil 0 red staining. 
Images covering the entire liver tissue area were taken and analyzed by a laboratory technician who was blind to the grouping. The collagen areas on Masson's trichrome staining images and the lipid areas on Oil 0 red staining images were assessed using ImageJ.

\section{Western blotting analysis}

Liver tissue lysates were prepared using radioimmunoprecipitation assay buffer (Sigma) supplemented with phosphatase and protease inhibitors (Sigma) per the manufacturer's instruction, subjected to $10 \%$ sodium dodecyl sulfate-polyacrylamide gel electrophoresis, and electroblotted onto polyvinylidene fluroride membranes. The Akt phosphorylation was detected using rabbit anti-phospho-Akt (Thr308) (Cell Signaling) and horseradish peroxidaseconjugated secondary antibodies (Amersham). After stripping using striping buffer (Thermo Scientific), total Akt levels were detected using rabbit anti-AKT (Santa Cruz). The protein bands were visualized with chemiluminescence reagent (Amersham) and quantitated as previously described. ${ }^{(14)}$

\section{Statistics}

All data were expressed as mean \pm SEM unless specified otherwise. Statistical tests were performed using one-way analysis of variance (ANOVA) with Bonferroni correction or unpaired $t$-test using GraphPad Prism (version 8.0.2; GraphPad Software). The significance level was set at * $\mathrm{p}<0.05$.

\section{Results}

\section{Characterization of WS-DEP and WIS-DEP}

The WS-DEP and WIS-DEP solutions were prepared in a concentration equivalent to that of $2 \mathrm{mg} / \mathrm{ml}$ DEP as shown in Figure 1A. Figure 1B shows the representative images of PBS, DEP, WS-DEP, and WIS-DEP. It was noted that WIS-DEP was opaque and indistinguishable from DEP, whereas WS-DEP was transparent but slightly different from PBS in color. Freeze-drying and weighing of WS-DEP and WIS-DEP revealed that DEP consisted of $18 \%$ WS-DEP and $82 \%$ WIS-DEP. The elemental compositions of WS-DEP and WIS-DEP were determined using inductively coupled plasma mass spectrometry as previously described(7) and presented on Table 1. Our analysis showed that almost all $P$ and $S$ of DEP were soluble in PBS; Al, Si, and Fe of DEP were partially soluble; and almost all other metals of DEP appeared to be insoluble in PBS (Table 1).

\section{Pulmonary effects of long-term instillation of WS-DEP or WIS-DEP}

To determine the individual roles of WS-DEP and WIS-DEP in the development of adverse health effects following DEP exposure, C57BI/6J mice were consecutively instilled with PBS, DEP, WS-DEP, and WIS-DEP for 15 weeks (Figure 1A). Figures 1C and 1D reveal that compared to instillation of PBS, instillations of DEP, WS-DEP, or WIS-DEP did not significantly influence the murine body weight and food intake. Body composition analysis after euthanasia showed that compared to instillation of PBS, instillation of either DEP or WIS-DEP, but not WS-DEP, significantly increased the weight of lung (Table 2). All other major organs of DEP, WS-DEP, and WIS-DEP-instilled mice had a weight comparable to that of PBS-instilled mice (Table 2). Histological analysis of the lung demonstrated a remarkable deposition of black carbon in the lung of DEP or WIS-DEP-instilled mice but not in that of PBS or WS-DEP-instilled mice (Figure 1E). Notably, all black carbon was apparently engulfed by macrophages (intracellularly located) and in the alveolar air space only (Figure 1E). We did not note any black carbon in the alveolar wall (Figure 1E). Inflammation scoring analysis revealed that versus instillation of PBS, instillation of either DEP or WIS-DEP, but not WS-DEP, significantly increased the pulmonary inflammation score (Figure 1F), primarily due to an increase in alveolar macrophages (Figure 1E and Table 3). The BALF cell number is another well-known indicator for pulmonary 
inflammation.(14) Figure 1G shows that compared to instillation of PBS, instillation of either DEP or WIS-DEP, but not WS-DEP, significantly increased the BALF cell count. To further document the pulmonary inflammatory response, we assessed the expression of pro-inflammatory cytokine mRNAs in the lung. Figures $\mathbf{1 H}-\mathbf{1} \mathbf{J}$ reveal that versus instillation of PBS, instillation of DEP significantly increased the pulmonary expression of TNFa, IL $-1 \beta$, and IL- 6 mRNAs, instillation of WIS-DEP significantly increased the pulmonary expression of TNFa and IL-1 $\mathrm{mRNAs}$, and in contrast, instillation of WS-DEP did not significantly increase any of these pro-inflammatory cytokine mRNAs in the lung.

\section{Blood effects of long-term instillation of WS-DEP or WIS-DEP}

Circulating pro-inflammatory cytokines are believed to play a crucial role in the development of extra-pulmonary effect following exposure to particulate matter.(11) Therefore, we compared the effects of instillations of either WSDEP or WIS-DEP on circulating cytokines. Figures $2 \mathrm{~A}-2 \mathrm{C}$ show that versus instillation of PBS, instillation of either DEP or WIS-DEP significantly increased the circulating IL-1 $\beta$; instillation of WIS-DEP also significantly increased circulating IL-6; and in contrast, instillation of WS-DEP did not significantly influence any of these circulating proinflammatory cytokines. Particulate matter exposure was shown to impact the homeostasis of blood lipids.(15) However, our assessment showed that compared to instillation of PBS, instillations of DEP, WS-DEP, or WIS-DEP did not significantly influence the levels of circulating total triglycerides, total cholesterol, LDL cholesterol, and HDL cholesterol (Figures 2D-2G). Circulating ALT and AST levels are two frequently used biomarkers for hepatic steatosis, a disease believed to be impacted by PM exposure.(16) We thus also measured the serum ALT and AST levels of these instilled mice. Figures $\mathbf{2 H}$ and $2 \mathrm{I}$ reveal that compared to instillation of PBS, instillation of DEP significantly increased the circulating ALT and AST levels, instillation of WIS-DEP significantly increased the circulating ALT level (Figure 2H) and had a non-significant trend of increase in the serum AST level (Figure 2I), and in contrast, instillation of WS-DEP did not influence the circulating ALT and AST levels.

\section{Hepatic effects of long-term instillation of WS-DEP or WIS-DEP}

Given the above data showing that instillation of WIS-DEP but not WS-DEP significantly increased the circulating biomarkers of hepatic steatosis, histological analysis of the liver was performed. H\&E staining displayed that compared to the hepatocytes of PBS-instilled mice, the hepatocytes of DEP-instilled or WIS-DEP-instilled, but not WS-DEPinstilled, mice had markedly more vacuoles (Figures $3 A$ ). To determine whether this is an increase in lipid droplets, Oil 0 staining was performed. Figures $3 \mathrm{~A}$ and $3 \mathrm{~B}$ show that versus instillation of PBS, instillation of DEP or WIS-DEP, but not WS-DEP, significantly increased the lipid content of liver sections. To further test the effect of instillation of WIS-DEP on hepatic steatosis, we assessed the lipid content of these livers using commercially available kits. Figure 3D shows that compared to instillation of PBS, instillation of DEP or WIS-DEP, but not WS-DEP, had a marked non-significant trend of increase in hepatic lipid content. Interestingly, Figures $3 \mathrm{C}$ and $3 \mathrm{E}$ reveals that DEP, WS-DEP, or WIS-DEPinstilled mice versus PBS-instilled mice all had significantly higher cholesterol and collagen contents in the liver. Liver insulin resistance was shown to account for the systemic glucose intolerance following instillation of DEP.(14) Figures 3F and 3G display that versus instillation of PBS, instillation of DEP or WIS-DEP, but not WS-DEP, significantly decreased insulin-induced Akt phosphorylation in the liver.

\section{Glucose homeostasis effects of long-term instillation of WS-DEP or WIS-DEP}

Long-term instillation of DEP was shown to result in marked systemic insulin resistance and glucose intolerance, primarily due to hepatic insulin resistance.(14) Therefore, the present study also analyzed the systemic glucose homeostasis of these instilled mice. Figures 4A and 4B show that versus PBS-instilled mice, DEP or WIS-DEP- 
instilled but not WS-DEP-instilled mice had a significantly higher glucose intolerance. DEP or WIS-DEP-instilled mice versus PBS-instilled mice also had a significantly higher insulin resistance (Figures 4C and 4D).

\section{Effects of long-term instillation of WS-DEP or WIS-DEP on the gut microbiota}

Mounting studies have shown that PM exposure may impact the composition of gut microbiota,(7) an emerging crucial mediator of various pathogenesis, including type II diabetes mellitus and non-alcoholic fatty liver disease. We thus assessed the effect of long-term instillations of WS-DEP or WIS-DEP on gut microbiota. $62612 \pm 6701$ valid sequences/mouse were obtained after optimization as described in Methods, and of them, $99.89 \%$ were $401-450$ bps in length (Figure S1). These sequences were clustered into 7982 OTUs, and the distribution of these OTUs in-between 4 experimental groups was shown by a Venn diagraph (Figure S2). It showed that about a half of these OTUs were shared by all 4 experimental groups (Figure S2). The a-diversity estimators of gut microbiota, including ACE, Chao-1, Shannon, and Simpson are believed to be critical indictors for the well-being of gut microbiota.(7) Figures 5A and 5B show that the ACE and Chao-1 estimators, well-known indicators for gut microbiota richness, of PBS, DEP, WS-DEP, or WIS-DEP-instilled mice were comparable. In contrast, DEP, WS-DEP, or WIS-DEP-instilled mice versus PBSinstilled mice all appeared to have a decrease in the Shannon estimator and an increase in the Simpson estimator (both suggest a decrease in the gut microbiota diversity, Figures 5C and 5D), but only the effect of long-term instillation of WS-DEP on the Shannon estimator was significant. These results suggest that instillations of DEP, WS-DEP, or WISDEP had a limited effect on the composition of gut microbiota. In agreement with this, the 4 experimental groups were not clearly clustered in the PCA, PCOA and hierarchical clustering analysis (Figures S3 and S4). To further document the individual roles of WS-DEP and WIS-DEP in DEP instillation impacting the composition of gut microbiota, the intra- and inter-group dissimilarities in gut microbiota composition were quantitated using the Bray-Curtis distance. Figure 5E reveals that the intra-group variations of DEP, WS-DEP, or WIS-DEP-instilled mice were comparable, but PBS-instilled mice had a markedly higher intra-group variation. In addition, the distances of DEP versus PBS, WSDEP versus PBS, and WIS-DEP versus PBS were remarkably bigger than the remaining three intergroup distances (Figure 5E), and by comparison, DEP versus WS-DEP had a smaller distance than that of DEP versus WIS-DEP.

The relative abundances of OTUs were presented on Table S1, and all the significantly differential OTUs identified by the LEfSe analysis were shown on Figure 6. Compared to PBS-instilled mice, DEP-instilled mice had 2 significantly over-represented phyla (Proteobacteria and Epsilonbacteraeota) and 1 significantly down-represented phylum (Deferribacteraceae), WS-DEP-instilled mice had 2 significantly over-represented phyla (Proteobacteria and Epsilonbacteraeota), whereas WIS-DEP-instilled mice had no significantly differentially represented phylum, suggesting that the effect of instillation of DEP on gut microbiota is attributable primarily to the water-soluble fraction (Figures 6A-6C). In addition, consistent with the above intergroup distance analysis, we noted that there were more significantly differential OTUs between DEP and WIS-DEP-instilled mice (Figure 6E) compared to those between DEP and WIS-DEP-instilled mice (Figure 6D). It is noteworthy that the over-representation of Proteobacteria in DEP or WSDEP-instilled mice versus controls was fully accounted for by the over-representation of Desulfovibrionales, the sulfate-reducing bacteria.

\section{Discussion}

To determine the individual roles of water-soluble and -insoluble fractions of $\mathrm{PM}_{2.5}$ in the development of systemic health effects following $\mathrm{PM}_{2.5}$ exposure will not only advance our understanding of the mode of action of $\mathrm{PM}_{2.5}$ but also may help develop more efficient pollution prevention policies. To our best knowledge, the present study is the first one to compare the systemic health effects of chronic instillations of water-soluble and -insoluble fractions of DEP in careful consideration of dosing and thus makes it possible to determine their individual contributions to the 
development of various health effects due to chronic instillation of DEP, a major source of urban ambient $\mathrm{PM}_{2.5}$. The major findings of this study include that 1) chronic instillation of the water-insoluble fraction but not the watersoluble fraction mimicked the effects of chronic instillation of DEP on pulmonary and systemic inflammations, hepatic lipid content and sensitivity to insulin, and systemic glucose homeostasis; 2) both chronic instillations of the watersoluble and -insoluble fractions mimicked the effects of chronic instillation of DEP on liver collagen and cholesterol contents; 3 ) although both the water-soluble and -insoluble fractions contributed to the effect of chronic instillation of DEP on the gut microbiota, the water-soluble fraction appeared to play a bigger role in this effect.

$\mathrm{PM}_{2.5}$ exposure directly targets the lung, and the inflammatory response of lung to $\mathrm{PM}_{2.5}$ exposure is widely believed to play a crucial role in not only the development of pulmonary injury induced by $\mathrm{PM}_{2.5}$ exposure but also many extrapulmonary health effects due to $\mathrm{PM}_{2.5}$ exposure, e.g., hepatic steatosis and insulin resistance.(11) We recently showed that the water-insoluble but not water-soluble fraction is responsible for the development of pulmonary inflammation due to acute instillation of DEP.(17) The present study revealed that the pulmonary inflammation induced by chronic instillation of DEP was also attributable to the water-insoluble fraction. Our results are consistent with several previous rodent model studies showing that acute pulmonary inflammatory response to ambient $\mathrm{PM}_{2.5}$ is primarily attributable to its water-insoluble fraction.(18-21) Notably, there are also two previous rodent model studies showing that the water-soluble fraction of residual oil fly ash (ROFA) or ambient $\mathrm{PM}_{2.5}$ provokes marked pulmonary inflammation.(22, 23) Unfortunately, because both studies did not have a ROFA or $\mathrm{PM}_{2.5}$ control and the water-soluble fraction was prepared using acid, it is impossible to compare their results with the present data. Our results are also consistent somehow with many cultured cell studies showing that the water-insoluble but not -soluble fraction accounts for the cytotoxicity and pro-inflammatory action of DEP or $\mathrm{PM}_{2.5}$.(24-29) Along with the putatively central role of pulmonary inflammation in the development of adverse health effects due to acute or chronic $\mathrm{PM}_{2.5}$ exposure,(11) the present study adds largely to the evidence that the water-insoluble fraction is more harmful than the water-soluble fraction.

The recent disease burden analysis revealed that exposure to $\mathrm{PM}_{2.5}$ significantly increases the mortality due to type 2 diabetes.(1) The present study showed that the insulin resistance and glucose intolerance induced by chronic instillation of DEP were fully accounted for by the water-insoluble but not water-soluble fraction of DEP. To our best knowledge, this is the first study investigating the individual roles of DEP fractions in the development of abnormal glucose homeostasis following chronic DEP instillation. This demonstration of the water-insoluble fraction as the primary culprit for the abnormal glucose homeostasis due to chronic DEP exposure will not just benefit the development of more focused pollution control policy but also sheds some light on the mechanism for the induction of abnormal glucose homeostasis by $\mathrm{PM}_{2.5}$ exposure. It is particularly noteworthy that the present study demonstrated that both abnormal glucose homeostasis and pulmonary inflammation induced by instillation of DEP were fully accounted for by the water-insoluble fraction. This is consistent with the notion that pulmonary inflammation is central in the development of adverse health effects following $\mathrm{PM}_{2.5}$ exposure(11) and thus strongly support preventing $\mathrm{PM}_{2.5}$ exposure-associated diabetes morbidity and mortality through targeting the water-insoluble fraction of $\mathrm{PM}_{2.5}$ and/or pulmonary inflammation.

In the research on $\mathrm{PM}_{2.5}$ pollution, there is a prevalent opinion that the chemical composition of $\mathrm{PM}_{2.5}$ is a major driver of resultant health effects including glucose intolerance. ${ }^{(3,11)}$ However, despite the well-known huge temporospatial variation in the chemical composition of ambient $\mathrm{PM}_{2.5},{ }^{(11)}$ epidemiological studies conducted worldwide have collectively shown that no any ambient $\mathrm{PM}_{2.5}$ can yet be dismissed as being unequivocally not associated with adverse health effects. ${ }^{(3,11)}$ In line with these epidemiological studies, we showed that ambient $\mathrm{PM}_{2.5}$ from Baltimore, Maryland, (5) Tuxedo, New York, ${ }^{(6)}$ and Shanghai, China ${ }^{(7)}$ had distinct chemical compositions, representing those of ambient 
$\mathrm{PM}_{2.5}$ in urban, rural, and construction areas, respectively, but exposures to these $\mathrm{PM}_{2.5}$ all elicited glucose intolerance. (7-10) These published epidemiological and toxicological studies strongly suggest that the development of glucose intolerance following $\mathrm{PM}_{2.5}$ exposure is either not strictly $\mathrm{PM}_{2.5}$ chemical composition-dependent or even $\mathrm{PM}_{2.5}$ chemical composition-independent. Given the universal pro-inflammatory action of water-insoluble particles, which may be accounted for by physical and morphological but not necessarily chemical characteristics,(30) our demonstration of the dependency of DEP instillation-induced glucose intolerance on the water-insoluble fraction of DEP indeed provides a potential mechanistic interpretation for the apparent independency of $\mathrm{PM}_{2.5}$ exposure-induced glucose intolerance on $\mathrm{PM}_{2.5}$ chemical composition.

We previously showed that chronic instillation of DEP elicited systemic insulin resistance and glucose intolerance primarily though induction of hepatic insulin resistance.(14) Zheng et al revealed that the hepatic insulin resistance induced by chronic $\mathrm{PM}_{2.5}$ exposure was coincident with a nonalcoholic fatty liver disease-like phenotype.(16) Interestingly, the present study demonstrated that both hepatic insulin resistance and accumulation of lipids in liver following chronic instillation of DEP were fully accounted for by the instillation of water-insoluble fraction. This is consistent with the abovementioned essential role of the water-insoluble fraction in the development of abnormal glucose homeostasis and once again raises the significance of targeting the water-insoluble fraction to prevent $\mathrm{PM}_{2.5}$ exposure-induced adverse health effects. However, it should be noted that the present study also showed that both instillations of the water-soluble or insoluble fraction of DEP significantly increased the hepatic contents of collagen and cholesterol, strongly suggesting that DEP exposure impacts the liver through both water-soluble and -insoluble fractions, albeit the water-insoluble fraction obviously plays a bigger role in this pathogenesis. It is well-known that the water-soluble fraction of $\mathrm{PM}_{2.5}$ readily enters the systemic circulation and thus highly likely impacts extrapulmonary organs directly, whereas the water-insoluble fraction of $\mathrm{PM}_{2.5}$ hardly enters the systemic circulation. Therefore, given that the circulating lipids were not significantly influenced by instillation of DEP or its water-insoluble fraction, further investigation is required to determine the circulating mediator(s) for the inhaled water-insoluble fraction of DEP impacting hepatic function and structure, which is widely believed to be egress from pulmonary inflammation but indeed not experimentally tested(11).

The gut microbiota is an emerging crucial player in various cardiometabolic diseases and is implicated in the development of adverse health effects due to chronic $\mathrm{PM}_{2.5}$ exposure.(31) However, in direct contrast to the abovediscussed dependency of DEP instillation-induced adverse health effects on its water-insoluble fraction, the present study showed that the effect of chronic DEP instillation on the gut microbiota is dependent primarily on its watersoluble fraction. This is evidenced by not just the group-group distance analysis showing that the WIS-DEP group versus the WS-DEP group had a bigger distance from the DEP group but also by the present data showing that DEPinstilled mice versus WIS-DEP-instilled mice had much more significantly differential OTUs than those between DEPinstilled mice and WS-DEP-instilled mice. Of these differential OTUs, Proteobacteria is particularly notable, as it is one of the two over-represented phyla in DEP-instilled mice versus PBS-instilled mice and this over-representation was fully accounted for by the water-soluble fraction of DEP. Furthermore, this over-representation of Proteobacteria in DEP-instilled mice was fully attributable to an increase in sulfate-reducing bacteria, and our analysis demonstrated that the water-soluble fraction contained all the sulfate of DEP. All these strongly suggest that the increase in gut sulfate-reducing bacteria may directly result from the administration of sulfate, providing an interesting example of modification of composition of gut microbiota by environmental factors. However, the pathogenic role of this significant modification of gut microbiota by WS-DEP remains to be determined. Since almost all tested adverse health effects following DEP instillation were attributable to WIS-DEP, this significant modification of gut microbiota by WS-DEP may play a trivial, if any, role only in the pathogenesis following DEP instillation. 


\section{Conclusion}

The present study demonstrates that the pulmonary and systemic inflammations, hepatic steatosis and insulin resistance, and systemic glucose intolerance following DEP instillation are all attributable to the water-insoluble fraction of DEP, providing a mechanistic interpretation for the apparent independency of $\mathrm{PM}_{2.5}$ exposure-induced glucose intolerance on $\mathrm{PM}_{2.5}$ chemical composition.

\section{Abbreviations}

DEP, diesel exhaust particles; $\mathrm{PM}_{2.5}$, fine particulate matter; WS-DEP, water-soluble fraction of DEP; WIS-DEP, waterinsoluble fractions of DEP; ROFA, residual oil fly ash; PBS, phosphate-buffered saline; IPGTT, intraperitoneal glucose tolerance test; ITT, insulin tolerance test; OUT, operational taxonomic unit; PCA, principal component analysis; PCOA, principal co-ordinates analysis; LEfSe, linear discriminant effect size; LDA, linear discriminant analysis; BALF, bronchoalveolar lavage fluid; AST, aspartate transaminase; ALT, alanine transaminase; LDL, low density lipoprotein;

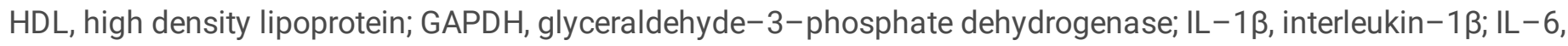
interleukin-6; TNFa, tumor necrosis factor alpha; ANOVA, analysis of variance.

\section{Declarations}

\section{Ethics approval and consent to participate:}

Fudan University is an AAALAC accredited institution. All procedures of this study were approved by the Institutional Animal Care and Use Committee (IACUC) at Fudan University, and all the animals were treated humanely and with regard for alleviation of suffering.

\section{Consent for publication:}

Not applicable.

\section{Availability of data and materials:}

All data generated or analysed during this study are included in this published article and its supplementary information files.

\section{Competing interests:}

The authors declare that they have no competing interests.

\section{Funding:}

This work was supported by the National Institutes of Health (R01ES024516 and R01ES032290 to ZY), the National Natural Science Foundation of China (Grant No. 91643205 to HK and Grant No. 82003414 and No. 81770805 to YX) and the National Key Research and Development Program of China (2019YFC1804503 to YX). All the funding bodies were not involved in the design of the study and collection, analysis, and interpretation of data and in writing the manuscript.

\section{Authors' contributions}

YX, ZL, YL, BP, RP, WS, WY, and MC acquired and analyzed the data used in the present study. YX, MC, HK, ZY, and YZ analyzed and interpreted the present results. $Y X, Z Y$, and $Y Z$ drafted the manuscript. MC and HK were also major 
contributors in writing the manuscript. All authors read and approved the final manuscript.

\section{Acknowledgements}

Not applicable.

\section{References}

1. Collaborators GBDRF. Global burden of 87 risk factors in 204 countries and territories, 1990-2019: a systematic analysis for the Global Burden of Disease Study 2019. Lancet. 2020;396(10258):1223-49.

2. Bowe B, Xie Y, Yan Y, Al-Aly Z. Burden of Cause-Specific Mortality Associated With PM2.5 Air Pollution in the United States. JAMA Netw Open. 2019;2(11):e1915834.

3. Wyzga RE, Rohr AC. Long-term particulate matter exposure: Attributing health effects to individual PM components. J Air Waste Manag Assoc. 2015;65(5):523-43.

4. Lippmann M. Toxicological and epidemiological studies of cardiovascular effects of ambient air fine particulate matter (PM2.5) and its chemical components: coherence and public health implications. Crit Rev Toxicol. 2014;44(4):299-347.

5. Chen M, Qin X, Qiu L, Chen S, Zhou H, Xu Y, et al. Concentrated Ambient PM2.5-Induced Inflammation and Endothelial Dysfunction in a Murine Model of Neural IKK2 Deficiency. Environ Health Perspect. 2018;126(2):027003.

6. Ying Z, Yue P, Xu X, Zhong M, Sun Q, Mikolaj M, et al. Air pollution and cardiac remodeling: a role for RhoA/Rhokinase. American journal of physiology Heart and circulatory physiology. 2009;296(5):H1540-50.

7. Wang W, Zhou J, Chen M, Huang X, Xie X, Li W, et al. Exposure to concentrated ambient PM2.5 alters the composition of gut microbiota in a murine model. Particle and fibre toxicology. 2018;15(1):17.

8. Sun Q, Zhang G, Chen R, Li R, Wang H, Jiang A, et al. Central IKK2 Inhibition Ameliorates Air Pollution-Mediated Hepatic Glucose and Lipid Metabolism Dysfunction in Mice With Type II Diabetes. Toxicological sciences : an official journal of the Society of Toxicology. 2018;164(1):240-9.

9. Wei W, Tang L, Chen M, Chen S, Zhou H, Ying Z. Intermittent fasting ameliorates PM2.5 exposure-induced abnormalities in glycaemic control. Toxicology and applied pharmacology. 2020;404:115181.

10. Hu Z, Chen M, Zhou H, Tharakan A, Wang X, Qiu L, et al. Inactivation of TNF/LT locus alters mouse metabolic response to concentrated ambient PM2.5. Toxicology. 2017;390:100-8.

11. NIH. Integrated Science Assessment (ISA) for Particulate Matter (Final Report, 2019). 2020.

12. Chen M, Liang S, Qin X, Zhang L, Qiu L, Chen S, et al. Prenatal exposure to diesel exhaust PM2.5 causes offspring beta cell dysfunction in adulthood. American journal of physiology Endocrinology and metabolism. 2018;315(1):E72-E80.

13. Xu Y, Wang W, Chen M, Zhou J, Huang X, Tao S, et al. Developmental programming of obesity by maternal exposure to concentrated ambient PM2.5 is maternally transmitted into the third generation in a mouse model. Particle and fibre toxicology. 2019;16(1):27.

14. Chen S, Chen M, Wei W, Qiu L, Zhang L, Cao Q, et al. Glucose Homeostasis following Diesel Exhaust Particulate Matter Exposure in a Lung Epithelial Cell-Specific IKK2-Deficient Mouse Model. Environ Health Perspect. 2019;127(5):57009.

15. Zhang T, Zheng Y, Gao Y, Zhao T, Guo S, Yang L, et al. Exposure to PM2.5 affects blood lipid levels in asthmatic rats through notch signaling pathway. Lipids Health Dis. 2019;18(1):160. 
16. Zheng Z, Xu X, Zhang X, Wang A, Zhang C, Huttemann M, et al. Exposure to ambient particulate matter induces a NASH-like phenotype and impairs hepatic glucose metabolism in an animal model. Journal of hepatology. 2013;58(1):148-54.

17. Tao S, Xu Y, Chen M, Zhang H, Huang X, Li Z, et al. Exposure to different fractions of diesel exhaust PM2.5 induces different levels of pulmonary inflammation and acute phase response. Ecotoxicology and environmental safety. 2021;210:111871.

18. Snow SJ, De Vizcaya-Ruiz A, Osornio-Vargas A, Thomas RF, Schladweiler MC, McGee J, et al. The effect of composition, size, and solubility on acute pulmonary injury in rats following exposure to Mexico city ambient particulate matter samples. J Toxicol Environ Health A. 2014;77(19):1164-82.

19. Ogino K, Zhang R, Takahashi H, Takemoto K, Kubo M, Murakami I, et al. Allergic airway inflammation by nasal inoculation of particulate matter (PM2.5) in NC/Nga mice. PloS one. 2014;9(3):e92710.

20. Donaldson K. Nonneoplastic lung responses induced in experimental animals by exposure to poorly soluble nonfibrous particles. Inhal Toxicol. 2000;12(1-2):121-39.

21. Zhao C, Niu M, Song S, Li J, Su Z, Wang Y, et al. Serum metabolomics analysis of mice that received repeated airway exposure to a water-soluble PM2.5 extract. Ecotoxicology and environmental safety. 2019;168:102-9.

22. Pardo M, Shafer MM, Rudich A, Schauer JJ, Rudich Y. Single Exposure to near Roadway Particulate Matter Leads to Confined Inflammatory and Defense Responses: Possible Role of Metals. Environmental science \& technology. 2015;49(14):8777-85.

23. Dreher K, Jaskot R, Kodavanti U, Lehmann J, Winsett D, Costa D. Soluble transition metals mediate the acute pulmonary injury and airway hyperreactivity induced by residual oil fly ash particles. Chest. 1996;109(3 Suppl):33S4S.

24. Leclercq B, Alleman LY, Perdrix E, Riffault V, Happillon M, Strecker A, et al. Particulate metal bioaccessibility in physiological fluids and cell culture media: Toxicological perspectives. Environmental research. 2017;156:148-57.

25. Vuong NQ, Breznan D, Goegan P, O'Brien JS, Williams A, Karthikeyan S, et al. In vitro toxicoproteomic analysis of A549 human lung epithelial cells exposed to urban air particulate matter and its water-soluble and insoluble fractions. Particle and fibre toxicology. 2017;14(1):39.

26. Shang Y, Wu M, Zhou J, Zhang X, Zhong Y, An J, et al. Cytotoxicity comparison between fine particles emitted from the combustion of municipal solid waste and biomass. Journal of hazardous materials. 2019;367:316-24.

27. Gali NK, Li G, Ning Z, Brimblecombe P. Diurnal trends in redox characteristics of water-soluble and -insoluble PM components. Environ Pollut. 2019;254(Pt A):112841.

28. Zou Y, Jin C, Su Y, Li J, Zhu B. Water soluble and insoluble components of urban PM2.5 and their cytotoxic effects on epithelial cells (A549) in vitro. Environ Pollut. 2016;212:627-35.

29. Omura S, Koike E, Kobayashi T. Microarray analysis of gene expression in rat alveolar epithelial cells exposed to fractionated organic extracts of diesel exhaust particles. Toxicology. 2009;262(1):65-72.

30. Klopfleisch R, Jung F. The pathology of the foreign body reaction against biomaterials. J Biomed Mater Res A. 2017;105(3):927-40.

31. Yang G, Wei J, Liu P, Zhang Q, Tian Y, Hou G, et al. Role of the gut microbiota in type 2 diabetes and related diseases. Metabolism. 2021;117:154712.

\section{Tables}

Table 1. The elemental compositions of PBS, DEP, WS-DEP, and WIS-DEP as determined by inductively coupled plasma mass spectrometry. Expressed as mean \pm SD of 3 independent assessments and in a unit of $\mu \mathrm{g} / \mathrm{l}$. ND, not detectable. 


\begin{tabular}{|c|c|c|c|c|}
\hline Elements & PBS & DEP & WS-DEP & WIS-DEP \\
\hline $\mathrm{Na}$ & $3036419.47 \pm 56914.79$ & $2965264.08 \pm 26252.92$ & $3311317.39 \pm 397701.10$ & $2934763.72 \pm 10039.88$ \\
\hline $\mathrm{Mg}$ & ND & $486.33 \pm 287.97$ & ND & $391.52 \pm 100.32$ \\
\hline Al & ND & $6266.90 \pm 172.79$ & $2556.10 \pm 383.36$ & $3414.36 \pm 155.12$ \\
\hline $\mathrm{Si}$ & ND & $3239.93 \pm 450.51$ & $2573.42 \pm 471.02$ & $2394.51 \pm 982.67$ \\
\hline$P$ & 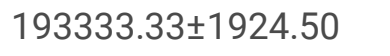 & $625000.00 \pm 21441.13$ & $620000.00 \pm 51532.08$ & $216666.67 \pm 1924.50$ \\
\hline S & ND & $12150.00 \pm 2547.08$ & $98900.00 \pm 10643.88$ & ND \\
\hline $\mathrm{Ti}$ & ND & $1786.16 \pm 1111.36$ & $32.00 \pm 23.26$ & $277.39 \pm 55.69$ \\
\hline $\mathrm{Ca}$ & ND & $1500.00 \pm 33.33$ & ND & $2016.67 \pm 160.58$ \\
\hline $\mathrm{Cr}$ & ND & $24.97 \pm 0.60$ & ND & $29.40 \pm 0.49$ \\
\hline $\mathrm{Mn}$ & ND & ND & ND & ND \\
\hline $\mathrm{Fe}$ & ND & $1747.82 \pm 208.99$ & $1049.70 \pm 347.12$ & $1161.58 \pm 199.97$ \\
\hline $\mathrm{Ni}$ & ND & ND & ND & ND \\
\hline $\mathrm{Cu}$ & ND & ND & ND & ND \\
\hline $\mathrm{Zn}$ & ND & $850.89 \pm 67.87$ & ND & $307.61 \pm 42.41$ \\
\hline As & ND & ND & ND & ND \\
\hline $\mathrm{Sr}$ & ND & ND & ND & ND \\
\hline $\mathrm{Ag}$ & ND & ND & ND & ND \\
\hline Sn & ND & ND & ND & ND \\
\hline $\mathrm{Ba}$ & ND & ND & ND & ND \\
\hline $\mathrm{TI}$ & ND & ND & ND & ND \\
\hline $\mathrm{Pb}$ & ND & ND & ND & ND \\
\hline
\end{tabular}

Table 2. The organ weighs of mice after the 15-week instillation of PBS, DEP, WS-DEP, or WIS-DEP. All data were presented as $\%$ of body weight (mean $\pm \mathrm{SD}$ ). ${ }^{\star} p<0.05$ versus PBS, one-way ANOVA. 


\begin{tabular}{|lllll|}
\hline Tissue & PBS & DEP & WS-DEP & WIS-DEP \\
\hline Brain & $1.58 \pm 0.03$ & $1.64 \pm 0.02$ & $1.53 \pm 0.11$ & $1.66 \pm 0.05$ \\
\hline Brown fat & $0.25 \pm 0.01$ & $0.28 \pm 0.02$ & $0.27 \pm 0.01$ & $0.29 \pm 0.02$ \\
\hline Heart & $0.53 \pm 0.02$ & $0.50 \pm 0.01$ & $0.48 \pm 0.01$ & $0.49 \pm 0.02$ \\
\hline Lung & $0.14 \pm 0.00$ & $0.16 \pm 0.01 *$ & $0.15 \pm 0.01$ & $0.16 \pm 0.01$ * \\
\hline Liver & $4.54 \pm 0.14$ & $4.65 \pm 0.20$ & $4.48 \pm 0.09$ & $4.58 \pm 0.15$ \\
\hline Spleen & $0.25 \pm 0.02$ & $0.22 \pm 0.02$ & $0.22 \pm 0.01$ & $0.22 \pm 0.01$ \\
\hline Pancreas & $0.51 \pm 0.02$ & $0.51 \pm 0.03$ & $0.47 \pm 0.03$ & $0.52 \pm 0.01$ \\
\hline Kidney & $1.42 \pm 0.05$ & $1.35 \pm 0.03$ & $1.34 \pm 0.02$ & $1.35 \pm 0.06$ \\
\hline Perirenal fat & $0.59 \pm 0.08$ & $0.72 \pm 0.06$ & $0.75 \pm 0.09$ & $0.80 \pm 0.09$ \\
\hline Testis & $0.69 \pm 0.02$ & $0.70 \pm 0.02$ & $0.72 \pm 0.02$ & $0.70 \pm 0.01$ \\
\hline Epididymis & $0.27 \pm 0.01$ & $0.26 \pm 0.01$ & $0.28 \pm 0.02$ & $0.27 \pm 0.01$ \\
\hline Epididymal fat & $1.76 \pm 0.17$ & $2.01 \pm 0.11$ & $2.19 \pm 0.19$ & $2.25 \pm 0.23$ \\
\hline Subcutaneous fat & $0.66 \pm 0.07$ & $0.74 \pm 0.07$ & $0.73 \pm 0.07$ & $0.84 \pm 0.12$ \\
\hline Skeletal muscle & $1.59 \pm 0.04$ & $1.63 \pm 0.02$ & $1.60 \pm 0.05$ & $1.64 \pm 0.04$ \\
\hline
\end{tabular}

Table 3. Pulmonary inflammation scoring system and results. ${ }^{*} p<0.05$ versus PBS, one-way ANNOVA.

\begin{tabular}{|llllll|}
\hline & Scoring standard & PBS & DEP & WS-DEP & WIS-DEP \\
\hline Oedema & absence, 0; presence, 1 & $0.20 \pm 0.18$ & $0.20 \pm 0.18$ & $0.80 \pm 0.18$ & $0.17 \pm 0.15$ \\
\hline Hyperemia & absence, 0; presence, 1 & $0.40 \pm 0.22$ & $0.40 \pm 0.22$ & $0.50 \pm 0.22$ & $0.33 \pm 0.19$ \\
\hline Hemorrhage & absence, 0; presence, 1 & $0.40 \pm 0.22$ & $0.40 \pm 0.22$ & $0.25 \pm 0.19$ & $0.17 \pm 0.15$ \\
\hline $\begin{array}{l}\text { Macrophage } \\
\text { infiltration }\end{array}$ & $\begin{array}{l}\text { presence of every 10\% alveoli }= \\
1\end{array}$ & $0.00 \pm 0.00$ & $1.00 \pm 0.00 *$ & $0.00 \pm 0.00$ & $1.00 \pm 0.00 *$ \\
\hline $\begin{array}{l}\text { Interalveolar thickness } \\
\text { presence of every 10\% alveoli }=\end{array}$ & $0.00 \pm 0.00$ & $0.40 \pm 0.22 *$ & $0.00 \pm 0.00$ & $0.67 \pm 0.19 *$ \\
\hline Alveolar distortion & $\begin{array}{l}\text { presence of every 10\% alveoli }= \\
1\end{array}$ & $0.00 \pm 0.00$ & $0.20 \pm 0.18$ & $0.25 \pm 0.19$ & $0.17 \pm 0.15$ \\
\hline
\end{tabular}

\section{Figures}




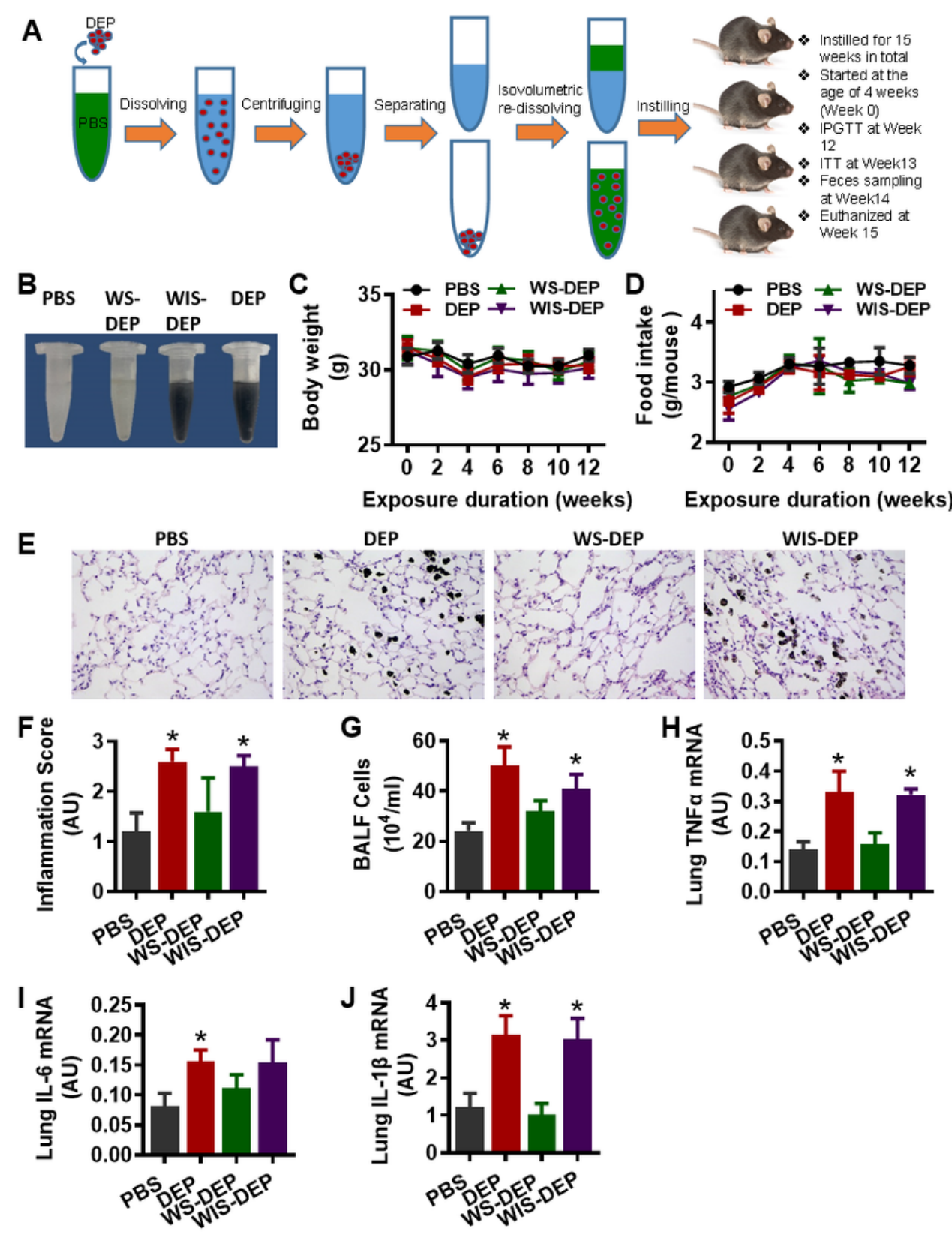

\section{Figure 1}

Pulmonary effects of long-term instillation of WS-DEP or WIS-DEP. A. The experimental scheme. B. Representative images of PBS, DEP, WS-DEP, and WIS-DEP. C and D. The murine body weight (C) and food intake (D) responses during the 15-week instillation. E and F. Representative images of murine lung sections after $\mathrm{H} \& \mathrm{E}$ staining (E) and the quantitation of murine pulmonary inflammation levels (F). G. The total cell number in BALF. H-J, Total RNA were extracted from murine lung and the indicated cytokine mRNAs were assessed by qPCR. $n=10 /$ group, * $p<0.05$ versus PBS, one-way ANOVA. 

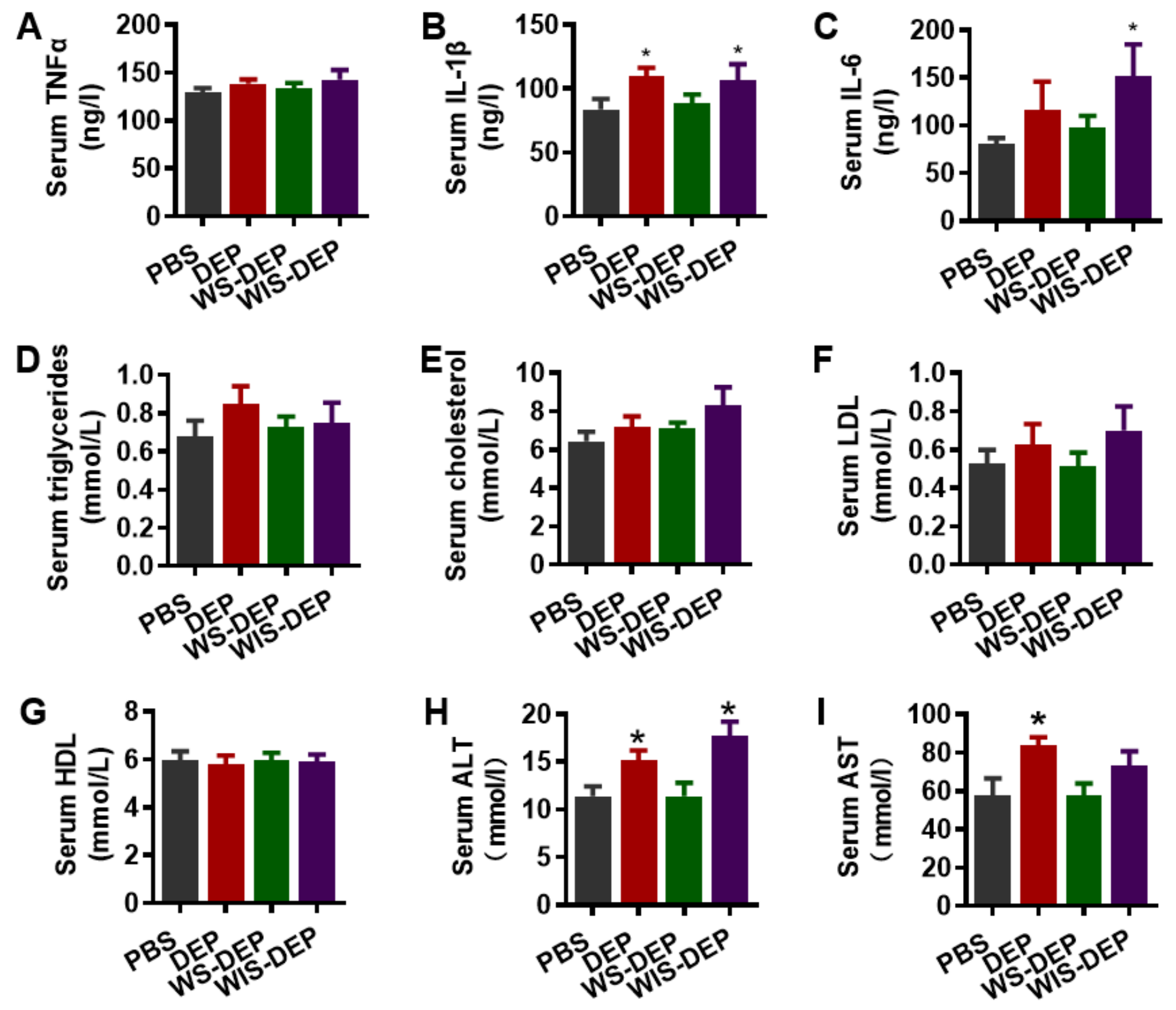

Figure 2

Blood effects of long-term instillation of WS-DEP or WIS-DEP. A-C. The indicated cytokines in serum were assessed using ELISA kits. D-G. Total triglycerides, total cholesterol, LDL cholesterol, and HDL cholesterol levels in serum were determined as described in Methods. H and I. ALT and AST levels in serum were obtained using commercially available kits as described in Methods. $n=10$ /group, ${ }^{*} p<0.05$ versus PBS, one-way ANOVA. 
A
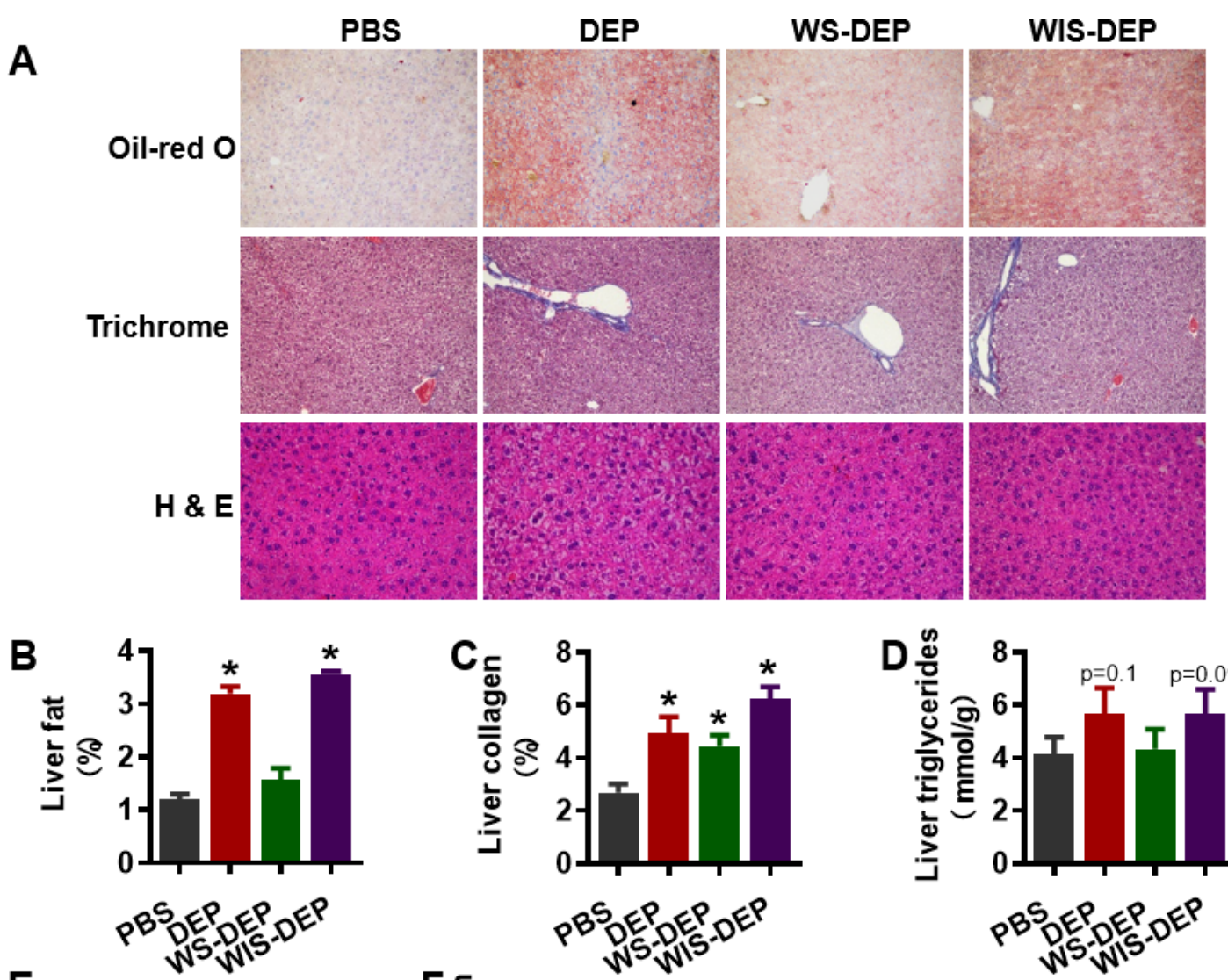

E

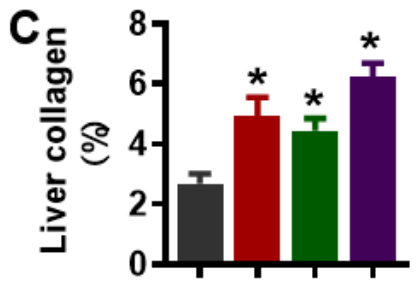

$\mathbf{F}$ 응
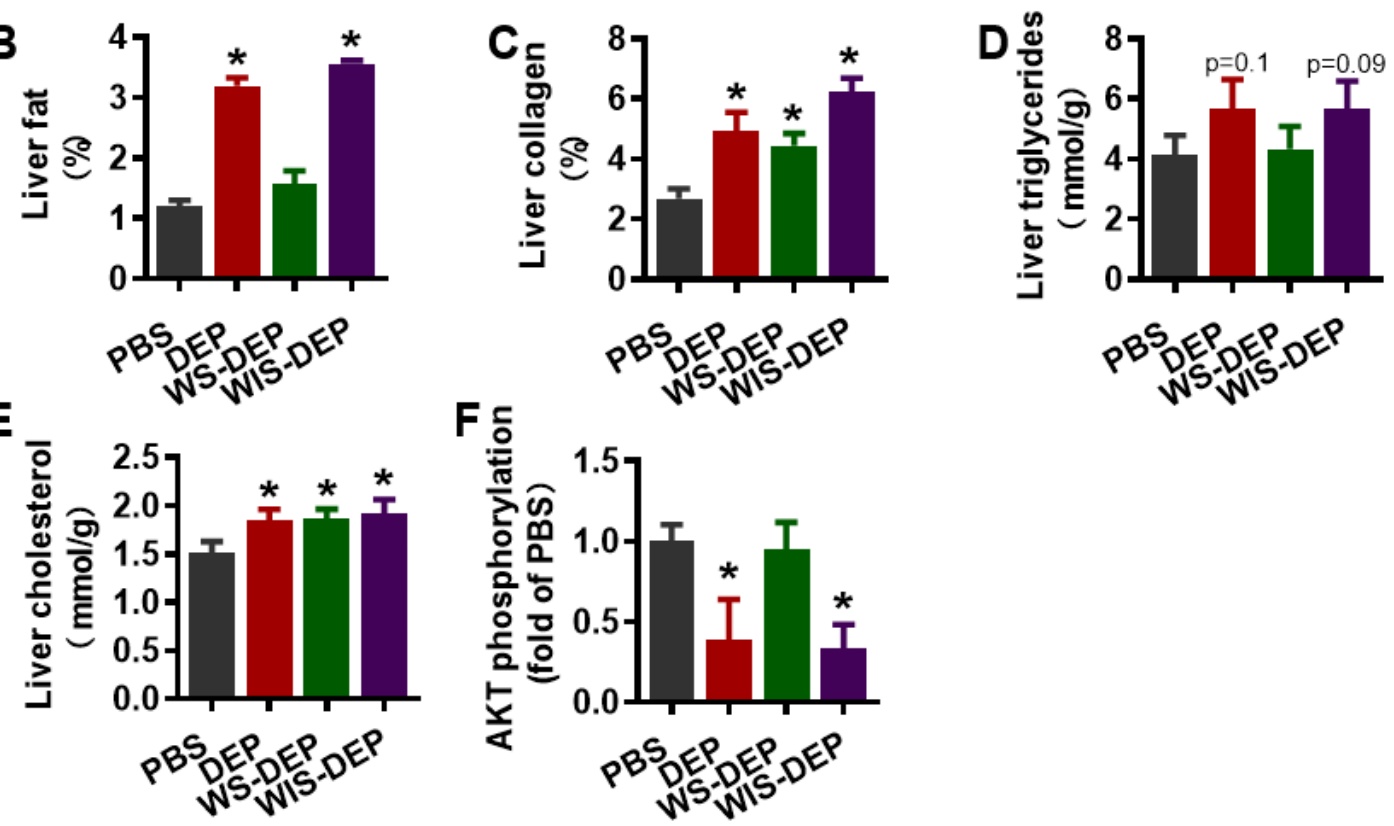

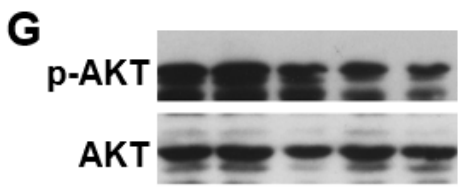

PBS

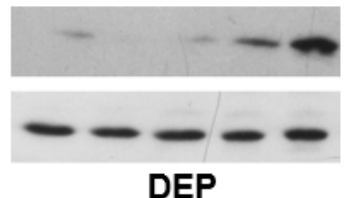

DEP

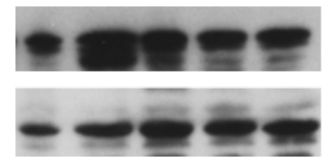

WS-DEP

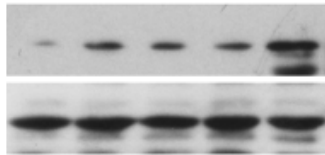

WIS-DEP

Figure 3

Hepatic effects of long-term instillation of WS-DEP or WIS-DEP. A. Representative images of murine liver sections subjected to Oil O red (upper), Masson's trichrome (middle), and H \& E staining (bottom). B. The quantitation of Oil $O$ red staining positive area on liver sections. C. The quantitation of collagen (blue) staining positive area on liver sections. D and E. Total triglycerides and total cholesterol levels in liver were determined using commercially available kits as described in Methods. $\mathrm{F}$ and $\mathrm{G}$. Akt phosphorylation levels in liver were determined by western blot analysis. $\mathrm{n}=$ $10 /$ group, ${ }^{*} p<0.05$ versus PBS, one-way ANOVA. 

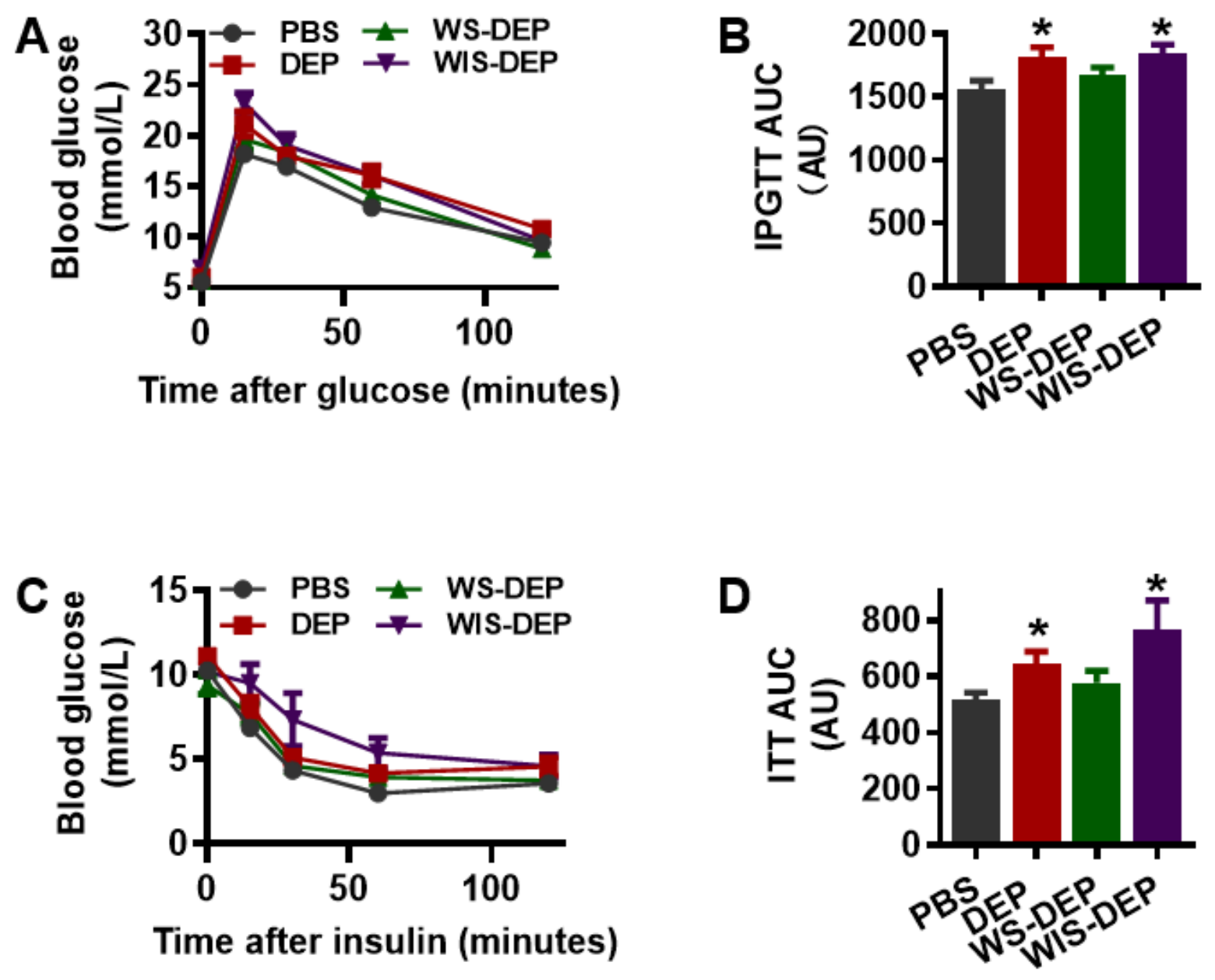

Figure 4

Effects of long-term instillation of WS-DEP or WIS-DEP on glucose homeostasis. A and B. IPGTT was performed after 12 weeks of instillation. The glycemic response curve (A) and area under curve (B) were presented. C and D. ITT was performed after 13 weeks of instillation, and the resultant glycemic response curve (C) and area under curve (D) were presented. $n=10 /$ group, ${ }^{*}<0.05$ versus PBS, one-way ANOVA. 

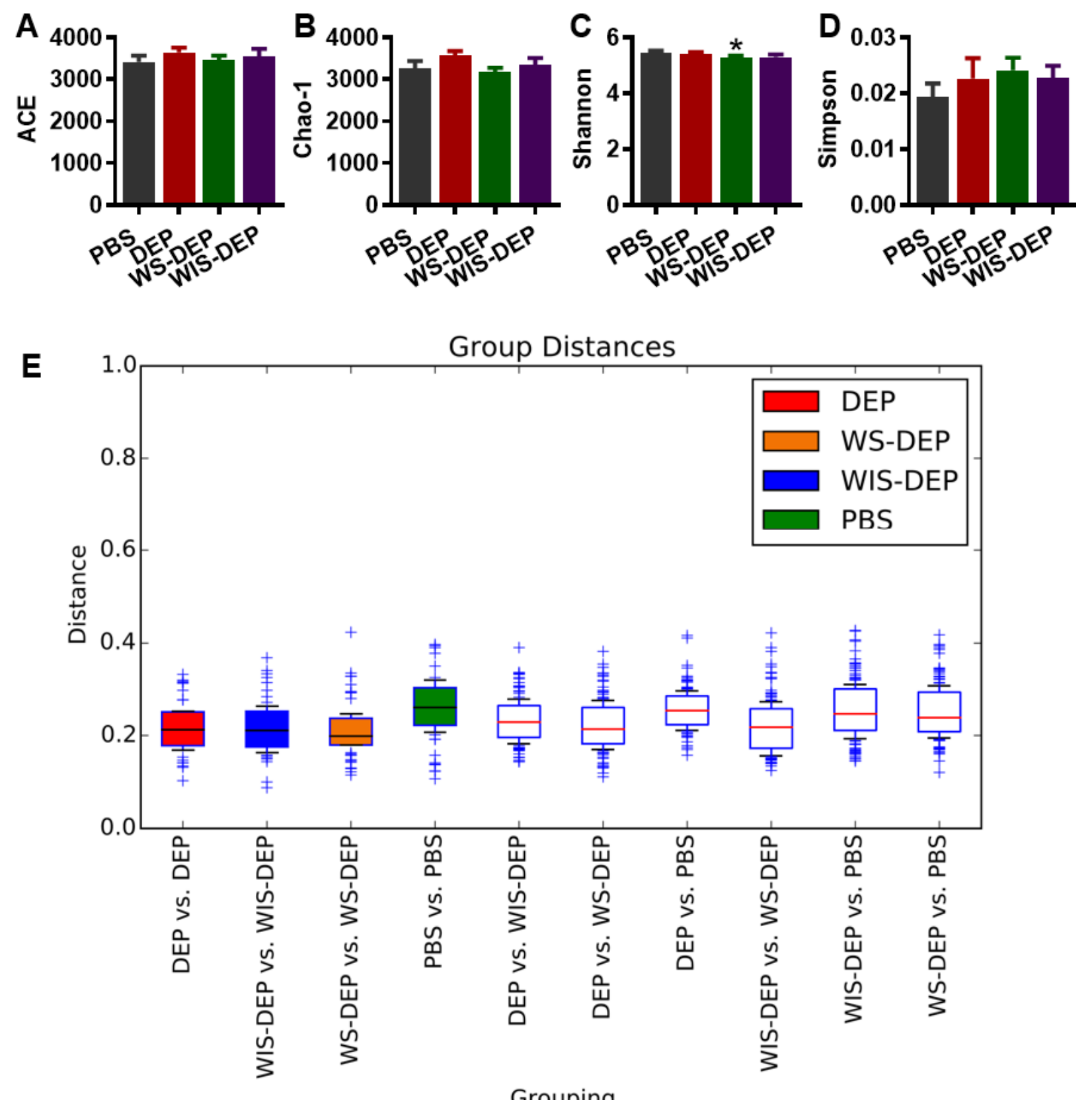

Grouping

Figure 5

Effects of long-term instillation of WS-DEP or WIS-DEP on gut microbiota. A-D. The a-diversity estimators including ACE, CHAO-1, Shannon, and Simpson were obtained as described in Methods and presented. E. The group-group distance analysis the Bray-Curtis dissimilarity analysis. $n=10$ /group, ${ }^{*} p<0.05$ versus PBS, one-way ANOVA. 
A

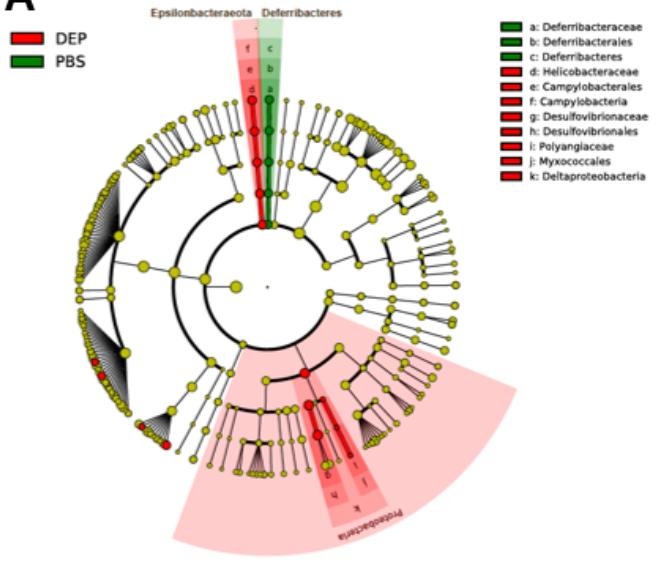

C $_{\text {PBS }}$
WIS-DEP

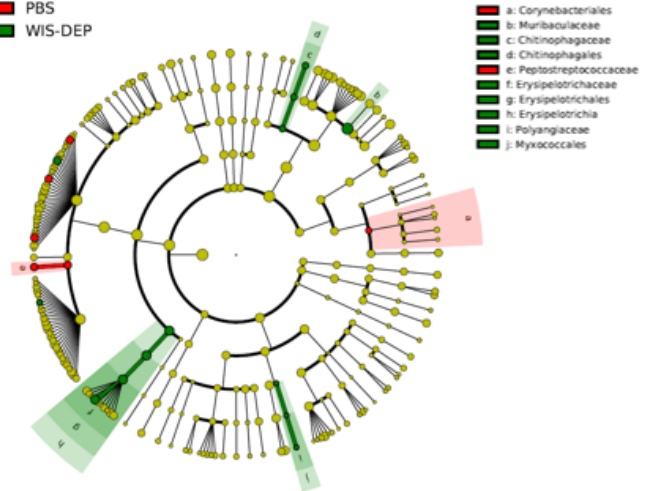

$\mathbf{E}$

ש WIS-DEP

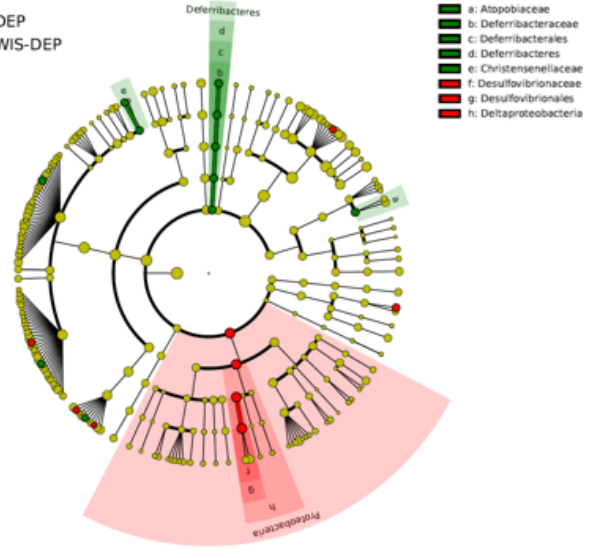

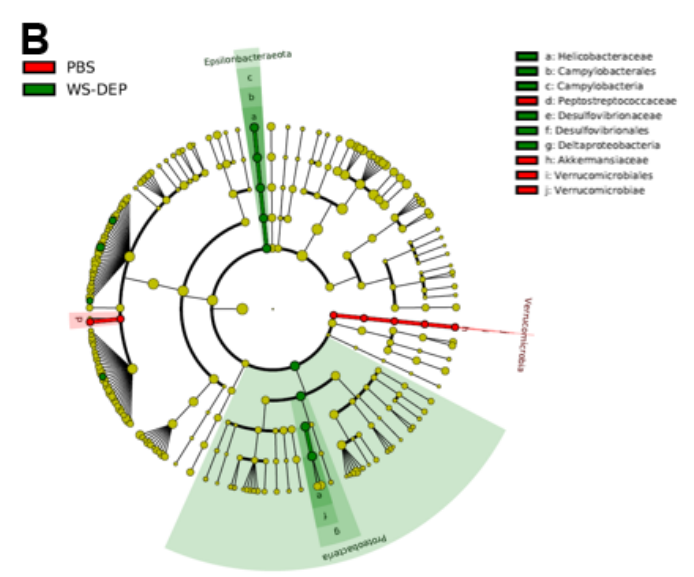

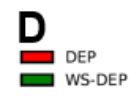
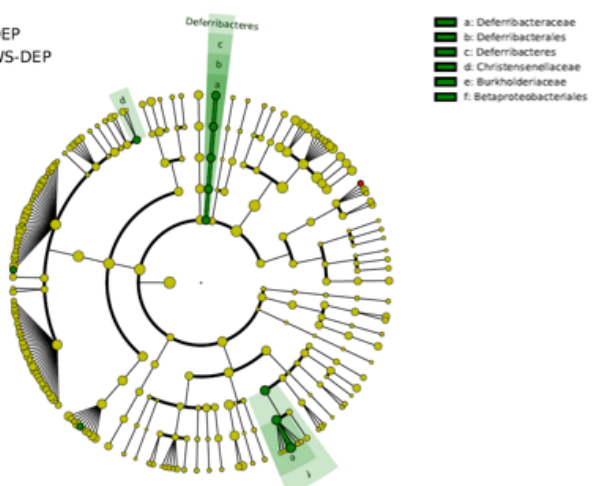

$\mathbf{F}$
שWIS-DEP
WS-DEP
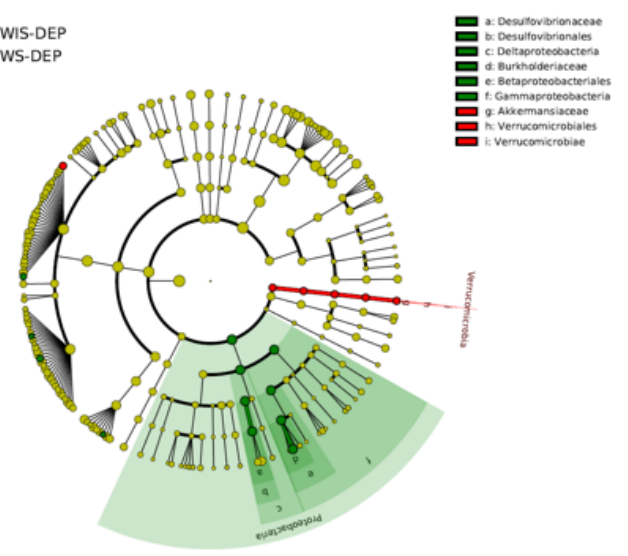

Figure 6

The differential represented OTUs. The relative abundances of OTUs were subjected to the LEfSe analysis, and significantly differential OTUs were presented using cladogram. $n=10 /$ group.

\section{Supplementary Files}

This is a list of supplementary files associated with this preprint. Click to download.

- SupplementaryFig.pptx

- SupplementaryTable1.docx 